\title{
A RELEVÂNCIA DE SOLUÇÕES ALTERNATIVAS PARA UNIVERSALIZAÇÃO DO ACESSO A ÁGUA NO BRASIL: O CASO DA SALTA-Z
}

\section{THE RELEVANCE OF ALTERNATIVE SOLUTIONS FOR UNIVERSALIZATION OF ACCESS TO WATER IN BRAZIL: THE CASE OF SALTA-Z}

\author{
Gustavo Carvalhaes Xavier Martins Pontual Machado ${ }^{(1)}$ \\ Professor Adjunto do Núcleo Interdisciplinar para o Desenvolvimento Social da Universidade Federal do Rio de Janeiro \\ (NIDES/CT/UFRJ), Pós-Doutorando em Saúde Pública e Meio Ambiente - ENSP/Fiocruz e Doutor em Psicossociologia \\ de Comunidades e Ecologia Social (EICOS/UFRJ). \\ Milena Manhães Rodrigues ${ }^{(2)}$ \\ Doutoranda em Turismo pela Universidade de São Paulo (EACH-USP), Mestra em Psicossociologia de Comunidades e \\ Ecologia Social (EICOS/UFRJ). Pesquisadora da Fundação Oswaldo Cruz (Fiotec -VPAAPS/Fiocruz). \\ Jaime Lopes da Mota Oliveira ${ }^{(3)}$ \\ Pesquisador em Saúde Pública do Departamento de Saneamento e Saúde Ambiental da Escola Nacional de Saúde Pública \\ Sérgio Arouca da Fundação Oswaldo Cruz (ENSP/Fiocruz). Mestre e Doutor em Microbiologia com ênfase em Ambiental \\ pela Universidade Federal do Rio de Janeiro (UFRJ) \\ Paulo Rubens Guimarães Barrocas ${ }^{(4)}$ \\ Pesquisador Titular do Departamento de Saneamento e Saúde Ambiental da Escola Nacional de Saúde Pública da \\ Fundação Oswaldo Cruz. Doutor em Oceanografia - Florida State University, com Mestrado em Geoquímica pela \\ Universidade Federal Fluminense (UFF). \\ Anna Cecília de Avelar Cortines ${ }^{(5)}$ \\ Agrônoma, com especialização em Ciências Ambientais e mestrado em Ciências Sociais pelo CPDA/UFRRJ. \\ Pesquisadora da Fundação Oswaldo Cruz (Fiotec -VPAAPS/Fiocruz)
}

\section{E-mail ${ }^{(1)}$ : gustavoxmartins@gmail.com}

\section{RESUMO}

Essa pesquisa teve como objetivo compreender a efetividade do processo de implementação da Solução Alternativa Coletiva Simplificada de Tratamento de Água com Zeólita (SALTA-z), a partir do olhar dos atores envolvidos, de quatro macrorregiões brasileiras, com diferentes contextos e níveis de adesão à tecnologia. A metodologia contemplou o levantamento documental, bibliográfico e entrevistas semiestruturadas. O estudo revelou os impactos positivos e os desafios com a operação da tecnologia. Ainda, foram compreendidas a aderência a SALTA-z vinculada ao pertencimento e a percepção de escassez de água de cada comunidade. Pode-se depreender que a cultura de cada território influi nos processos de educação, mobilização e participação social. Neste sentido, uma abordagem psicossocial, dialógica e inclusiva, é crucial para otimizar a efetividade de implementação e participação social em tecnologias de tratamento de água como a SALTA-z e assim promover mais ferramentas para garantir a universalização do acesso à água potável segura no país.

\begin{abstract}
The aim of this research is to identify the barriers and facilitators for an effective implantation of the Simplified Collective Alternative Solution for Water Treatment with Zeolite (SALTA-z), from the perspective of the actors involved from four Brazilian macro-regions, with different contexts and levels of adherence to the technology. The methodology included a literature review and semi-structured interviews. The study revealed the positive impacts and challenges with the operation of the technology. Furthermore, adherence to SALTA-z was linked to the belonging and the perception of water scarcity of each community. It can be inferred that the culture of each territory influences the processes of education, mobilization and social participation. In this sense, a psychosocial, dialogical and inclusive approach is crucial to optimize the effectiveness of implementation and social participation in water treatment technologies such as SALTA-z and thus promote more tools to ensure the universal access to safe drinking water.
\end{abstract}

Palavras-chave: Qualidade da Água. SALTA-z. Participação Social, Água para consumo humano. Saneamento.

Key words: Water Quality, Drinking water, Sanitation SALTA-z, social participation. 


\section{INTRODUÇÃO}

O acesso a água e ao saneamento são direitos humanos universais que estão relacionados ao ambiente sustentável, a promoção da saúde e, por conseguinte, a qualidade de vida, entre outros. No entanto, esse é um direito suprimido a grande parte da população que reside em áreas consideradas rurais ou em territórios tradicionais, com habitações remotas e afastadas de centros urbanos (BRASIL, 2019).

Esta carência deve-se a distância entre as casas, que ocasiona em uma dificuldade de instalação de sistemas convencionais de tratamento e distribuição de água. Ainda há falta de conhecimento sobre tecnologias existentes, de pessoal qualificado nos municípios e baixo fomento de recursos públicos em saneamento (SANTOS \& CARVALHO, 2018; BRASIL, 2017).

Diante desse cenário, a Superintendência Estadual da Funasa do Pará (Suest/PA) desenvolveu, em 2009, a tecnologia denominada "Solução Alternativa de Tratamento de Água por zeólita - SALTAz", solução coletiva para o suprimento de água em pequenas comunidades (BRASIL, 2017) para atender a realidade dos ribeirinhos. A SALTA-z é uma tecnologia desenvolvida para tratamento coletivo de água para consumo humano, de fácil instalação e funcionamento, com estrutura simples e utilização de filtro e dosadores com atributos artesanais, sendo de baixo custo de implantação e manutenção (SANTOS \& CARVALHO, 2018).

O projeto envolve cooperação técnica entre as Suest da Funasa com os municípios, a partir de repasse de recursos, transferência de tecnologia, capacitações e orientações técnicas referentes à implementação da SALTA-z (SANTOS \& CARVALHO, 2018).

A tecnologia tem tido boa aceitação na região Norte, até porque foi concebida para resolver os desafios inerentes aquele contexto específico. Ao ser replicada para outras regiões brasileiras surgiram diferenças quanto a aceitabilidade da tecnologia, cujos fatores precisam ser investigados, de forma a não comprometer a funcionalidade e objetivos da SALTA-z. Nesse sentido, o objetivo deste estudo foi avaliar a efetividade do processo de implementação da tecnologia "Solução Alternativa de Tratamento de Água por zeólita - SALTA-z", a partir de abordagem psicossocial dos atores locais, em comunidades rurais de quatro municípios das diferentes macrorregiões do Brasil - Norte, Nordeste, Sudeste e Sul.

A relevância deste trabalho se justifica pela baixa produção científica existente sobre a SALTA-z, demonstrando a necessidade de fortalecer a pesquisa, inclusive para propor melhorias e respaldar o uso da tecnologia, e evidenciando o ineditismo deste estudo e a necessidade de seu aprofundamento. Vale salientar que as publicações encontradas não têm como objetivo ou objeto a implantação da SALTA-z e seus respectivos impactos. Contudo, tais estudos discutem as limitações para adesão da tecnologia, ou ainda o reconhecimento acadêmico quanto a sua pertinência e capacidade de desempenho.

Olaerts (2013) reconhece os benefícios da SALTA-z para o saneamento básico no quilombo Cantão das Lombas, indicando tal tecnologia como solução alternativa de tratamento de água para a comunidade. $\mathrm{O}$ trabalho argumenta que no contexto da comunidade, é interessante analisar sistemas alternativos de tratamento de água para remoção de ferro e manganês, destacando a modalidade SALTA-z, o que mostra um reconhecimento acadêmico da tecnologia e de suas potencialidades. Na mesma vertente, a partir do levantamento das condições higiênico-sanitárias da comunidade Maiauatá (Igarapé-Miri - PA), Rodrigues et al (2018) propõem a implementação do projeto SALTA-z como exemplo de solução tecnológica de tratamento que garante água potável de qualidade, que estimula "a autonomia e a sustentabilidade dessas comunidades" (RODRIGUES et al, 2018, p.5). Defendem ainda que a adoção de tecnologias como essa, "não são onerosas tão pouco complexas, mas precisam ser engajadas e postas em prática" (RODRIGUES et al, 2018, p.7). 
Além do reconhecimento dos impactos positivos e da simplicidade da tecnologia, há estudos que demonstram as suas limitações, principalmente, com relação às questões de educação, mobilização, engajamento e participação social, que emergem muitas vezes das questões relacionadas à cultura hídrica de cada localidade. Neste cenário, o artigo de Santos et al (2020), referente ao abastecimento de água para consumo humano para os ribeirinhos de Sacaí (Caracaraí, RR), relata que embora a comunidade disponha do sistema SALTA-z desde 2019, observa-se que o consumo da água do rio sem nenhum tratamento persiste. Outras críticas são feitas quanto ao alcance da tecnologia devido a inexistência de "rede de encanamento para levar água tratada às residências, o que exige uma readequação na cultura do uso da água" (SANTOS et al, 2020, p.55). Os autores sugerem ainda que o consumo da água do rio deve-se a sua proximidade das habitações e o uso desta fonte como prática de cerca de quatro décadas na comunidade. Este ponto denota a importância da cultura hídrica e da percepção de risco da população relativa ao consumo de água insegura, que por sua vez impactam nos seus hábitos salutares e na sustentabilidade de qualquer solução alternativa. Em consonância, o artigo de Seabra (2019) também aponta a inexistência de rede de distribuição como desafio para aceitabilidade da tecnologia. O texto cita vários povoados/comunidades com SALTA-z, indicando sempre o mesmo problema: a falta de rede de distribuição e o hábito de consumir água direto do rio que está mais próxima aos domicílios.

Vale ressaltar que, assim como estabelecido no Programa de Saneamento Brasil Rural (PSBR), ações estruturais devem estar alinhadas com ações estruturantes a fim de que as intervenções sejam perenes e sustentáveis (BRASIL, 2019). Nesse sentido, o presente estudo buscou conhecer as realidades de diversas comunidades que receberam a SALTA-z, para compreender a efetividade da implementação dessa tecnologia. Esse estudo buscou compreender a participação social a partir da percepção de cultura hídrica, para assim otimizar a universalização do acesso a água segura no Brasil, especialmente no contexto rural, onde há maior vulnerabilidade da população.

\section{METODOLOGIA}

Partindo do pressuposto que o indivíduo é o sujeito em perspectiva, influenciado pelo contexto no qual está inserido e ao mesmo tempo agente transformador do mesmo, faz-se necessário analisar a percepção das populações sobre as tecnologias. Somente deste entendimento, a partir dos territórios, pode-se compreender, de fato, como tem se dado essa aceitabilidade e integração na prática. A psicossociologia contribui como perspectiva da pesquisa na medida em que envolve a compreensão das relações e representações sociais dos grupos e a configuração de subjetividades, neste caso, com o viés da saúde ambiental (MOSCOVICI, 1985).

Diante disso, a metodologia, de natureza qualitativa, envolveu levantamento bibliográfico e documental e trabalho de campo em comunidades rurais de quatro municípios onde foram implantadas a SALTA-z. Foram escolhidas comunidades com diferentes contextos e nível de adesão à tecnologia buscando compreender as diferenças psicossociais nestes contextos diversos, consistindo em: duas experiências de alta adesão (Rio do Oeste - SC e Limoeiro do Ajuru - PA), uma experiência em processo de adesão (Mamanguape - PB) e uma experiência de conflito (Comunidade Remanescente de Quilombo Boa Esperança, Areal - RJ), com falta de adesão e ruídos na comunicação entre os atores locais.

As informações foram coletadas por meio de observação participante e entrevistas semiestruturadas realizadas individualmente ou em grupos focais, de 30 de abril a 10 de dezembro de 2020. Foram entrevistados 50 atores locais, que participaram do processo de implementação e operacionalização da tecnologia: 7 servidores das Suest/Funasa, por serem responsáveis pelo desenvolvimento e articulação para implantação da tecnologia; 14 gestores/técnicos municipais e 4 agentes comunitários de saúde, parceiros na iniciativa; 8 operadores comunitários da tecnologia, responsável pela operação e manutenção da tecnologia na comunidade; e 17 comunitários/lideranças locais, beneficiários da 
tecnologia. Todavia, para fins deste resumo expandido, optou-se pelo recorte do estudo pelo olhar apenas dos entrevistados das Suest/Funasa, utilizando a análise interpretativa das narrativas como técnica para composição dos resultados (MORIN, 2004).

\section{RESULTADOS E DISCUSSÃO}

A partir das entrevistas com representantes das quatro Suest (SC, PA, PB e RJ), que atuam diretamente com a implementação da SALTA-z nas comunidades rurais e tradicionais, foi possível identificar fatores que podem ser indicativos de maior ou menor adesão e engajamento da população beneficiária à esta tecnologia e articular estes dados com reflexões e questões levantadas nas entrevistas em campo com os atores locais.

As comunidades estudadas partem de uma situação de fornecimento de água fora dos padrões de potabilidade para o consumo humano, no entanto, diferem quanto a condição de acesso à água, o que constitui um outro fator de aceitabilidade e engajamento da SALTA-z, segundo os entrevistados.

Em relação as entrevistas com os 7 participantes representantes da SUEST foram levantadas considerações recursivas, em especial, a Educação em Saúde como fator essencial de maior ou menor adesão e engajamento da população, entre outros indicativos: a necessidade de elaborar e conduzir orientações com metodologia adequada a distância, principalmente diante do contexto de distanciamento social imposto pela Covid-19; o envolvimento do setor de educação da Funasa no acompanhamento da implementação da SALTA-z junto aos municípios, para a construção de um plano de educação e saúde; a implicação da cultura de cada território que infere na resistência ao uso do cloro para desinfecção, especialmente na área rural; o monitoramento da água e dos impactos da SALTA-z na qualidade de vida das populações atendidas; a imprescindibilidade da atuação intersetorial e do comprometimento das equipes e lideranças comunitárias; a condição prévia de acesso à água, para compreender a vontade real de cada comunidade em receber a tecnologia; e ainda, a relação entre expectativas/conquistas de serviços hídricos.

Como sugestões, este grupo apontou a relevância: do alinhamento entre prefeitura e comunidade quanto à responsabilidade sobre a gestão e reposição dos insumos necessários a operação da SALTA$\mathrm{z}$, a ser definido de acordo com as possibilidades dos dois entes, em articular conteúdo sobre a tecnologia na formação dos agentes comunitários de Saúde (ACS) e elaboração/disponibilização de projeto de estrutura (base) para instalação desta.

As considerações elencadas levaram em conta as perspectivas dos entrevistados quanto a situação precedente e atual da SALTA-z relativa às condições de acesso a água em cada território (no respectivo estado da Suest que atuam).

Por fim, segundo representantes das Suest, o comprometimento das equipes e lideranças comunitárias envolvidas tem um papel importante na aceitabilidade da SALTA-z pelas comunidades. Nessa relação em que o sucesso da SALTA-z depende muito da gestão feita pelo município e pela comunidade beneficiada, a Funasa lida com as variáveis de engajamento dos prefeitos, técnicos da vigilância ambiental/da água e da própria comunidade, sendo que as configurações dessas variáveis mudam de acordo com cada município. Há casos de um bom engajamento do prefeito e do técnico da prefeitura e comunidade, como acontece em Limoeiro do Ajuru e Rio do Oeste, gerando pertencimento e autonomia e, por conseguinte, efetividade na implementação. Enquanto em Mamanguape a ação da prefeitura, mesmo bem-intencionada, tende ao assistencialismo, aliada a competição interna com outro acesso a água transportado da cidade até uma caixa d'água instalada pela prefeitura, tornam a tecnologia secundária e a operação dependente. Já em Areal, o engajamento tanto do município quanto da comunidade quilombola apresenta fragilidade, assentada em comunicação com ruído e resistência a adesão por histórico de conflitos fundiários e solicitações não atendidas da comunidade - se comparada a expectativa por um sistema de tratamento e distribuição de água encanada 
individualizada e a realidade de implantação de um sistema coletivo e de chafariz (que exige coleta e operação).

Pode-se constatar a importância de uma atuação intersetorial, que envolva tanto uma prefeitura engajada, quanto uma comunidade incluída no processo, que compreenda os impactos positivos, mas também os possíveis efeitos adversos, inclusive referentes a manutenção do funcionamento das instalações e o cuidado com os reagentes envolvidos no processo de tratamento.

$\mathrm{O}$ estudo realizado com as equipes das Suest da Funasa demonstrou a preocupação e engajamento dos técnicos com a implantação da tecnologia e ao mesmo tempo a sua fragilidade e insuficiência, pela necessidade de uma atuação intersetorial e de, tanto a prefeitura, quanto a comunidade estarem mutuamente implicadas no processo. Assim, a educação e participação social mostram-se como estruturantes para um real engajamento de todas as partes (Brasil, 2019). Esses pontos também foram verificados na prática nos campos estudados.

Essas situações nos levam a refletir sobre a relação entre expectativas/conquistas de serviços e o que a política pública oferece. Em outras palavras, a política pública precisa dialogar com a população a ser beneficiada, tratando-a como sujeito, e considerando as condições em que ela vive e quais melhorias ela busca em determinados serviços. As adequações e complementações na SALTA-z, podem gerar um balanço positivo nessa relação. Por outro lado, propor uma tecnologia, que a despeito de melhorias na qualidade da água, venha a trazer de volta uma condição anteriormente superada, como ter que coletar água em outro local e carregar até sua casa ${ }^{1}$, pode significar um balanço negativo nessa relação. Os estudos de Santos et al. (2020) e Seabra (2019) também relatam a dificuldade de se instalar soluções coletivas em comunidades que os moradores já estão acostumados a ter água disponível dentro de suas moradias, mesmo que sabidamente impróprias para consumo humano.

Assim, essas reflexões têm que estar associadas a outros fatores de qualidade de vida e aos riscos do consumo de água impropria, que podem ser invisíveis a essas populações, como é o caso da necessidade de tratamento da água e os benefícios associados. Compreende-se, desta forma, que as ações de educação em saúde são fundamentais e contribuem para o empoderamento das comunidades. As informações acima retratam a relevância de adequação sociotécnica a partir de diálogo entre Funasa - prefeitura - comunidades, para que ocorra efetividade na implementação da SALTA-Z como uma ferramenta de acesso ao direito universal a água.

\section{CONCLUSÕES}

Com essa pesquisa pode-se compreender a efetividade e as respectivas limitações do processo de implementação da Solução Alternativa Coletiva Simplificada de Tratamento de Água com Zeólita (SALTA-z), a partir do olhar dos atores envolvidos, para este resumo, especificamente representantes das Suest. Os resultados possibilitam que se tenham mais elementos para melhorar a implementação da tecnologia, podendo aperfeiçoar sua gestão para reaplicação com perenidade.

O estudo revelou necessidade de adequação sociotécnica na operação da tecnologia, especialmente na etapa de desinfecção, mas também as soluções propostas/implementadas nos territórios. Ainda, foram compreendidos os impactos positivos da utilização da tecnologia e a aderência vinculada também ao pertencimento e a percepção de acessibilidade/escassez de água de cada comunidade. Outro ponto verificado é como a cultura hídrica, que está relacionada a questão cultural e identitária de cada território, influi nos processos de educação, mobilização e participação social. Logo, a cultura de cada comunidade com relação a disponibilidade de água e as questões socioeconômicas implicam em diversos graus de adesão, participação e envolvimento com a tecnologia.

\footnotetext{
${ }^{1}$ Essa condição geralmente implica um esforço de trabalho e ocupação de tempo diário, geralmente das mulheres, que pode inclusive resultar em problemas de saúde.
} 
Assim, pode-se compreender a relevância da participação da comunidade para garantir efetividade da tecnologia. Ressalta-se, ainda, a importância de acompanhamento sistemático, de ter equipes para avaliação da tecnologia em campo, com as comunidades, conhecendo as pessoas e o que está sendo implementado. Essa relação de avaliação e monitoramento cria uma relação de troca, de confiança que se retroalimentam. Neste contexto, outras possibilidades de estudo relativo a SALTA-z poderiam compreender o entendimento de bem comum, aprofundado por Ostrom. Ainda, verifica-se a necessidade da condução de estudos quantitativos em escala piloto, para se conhecer e difundir a eficiência técnica da tecnologia.

Futuramente os dados obtidos com a comunidade serão tratados e publicados, apresentando desdobramentos da pesquisa, a partir do olhar dos atores locais. Por fim, o presente estudo, pode contribuir com a produção científica relativa ao campo de saúde ambiental, para otimizar a efetividade de implementação e participação social em tecnologias de tratamento de água como a SALTA-z e assim promover mais ferramentas para garantir a universalização do acesso à água potável no país.

\section{REFERÊNCIAS}

BRASIL. Ministério da Saúde. Fundação Nacional de Saúde. Manual da solução alternativa coletiva simplificada de tratamento de água para consumo humano em pequenas comunidades utilizando filtro e dosador desenvolvidos pela Funasa/Superintendência Estadual do Pará. Brasília: Funasa, 49 p. 2017. Disponível em:

http://www.funasa.gov.br/documents/20182/38937/Manual+da+SALTA-z+WEB.pdf/ae8139d420a6-46d0-acb6-10b9cea2b7b2

BRASIL. Ministério da Saúde. Fundação Nacional de Saúde. Programa Nacional de Saneamento Rural - $1^{a}$ edição. Brasília: Funasa. 2019. Disponível em:

http://www.funasa.gov.br/documents/20182/38564/MNL_PNSR_2019.pdf/08d94216-fb09-468e$\underline{\text { ac98-afb4ed0483eb }}$

MORIN, A. Pesquisa-ação integral e sistêmica: uma antropopedagogia renovada. Rio de Janeiro: DP\&A. 2004.

MOSCOVICI, S. (org.). Psicologia Social. Barcelona, Espanha: Paidós. 1985.

OLAERTS, L. Programa de saneamento básico em saneamento precário: estudo de caso do quilombo Cantão das Lombas. 100p. 2013. Trabalho de Conclusão de Curso (Graduação em Engenharia Ambiental) - Instituto de Pesquisas Hidráulicas e Escola de Engenharia, Porto Alegre, Universidade Federal do Rio Grande do Sul.

RODRIGUES, D. C., CONCEIÇÃO, A. C. S., SOUSA, L. P. A., CAXIAS, N. S. Análise de tecnologias para saneamento de comunidade ribeirinha na Amazônia: identificação de potencialidades e proposição de tecnologias. Anais: $13^{\circ}$ Seminário Nacional de Resíduos Sólidos, Cuiabá - MT. ABES - Associação Brasileira de Engenharia Sanitária e Ambiental. 2018.

SANTOS, E. R, LEAL, R. S., VERAS, A. T. R., MAIA, R. O. S. (2020). Geograficidade amazônica: a percepção do lugar dos ribeirinhos de Sacaí, Baixo Rio Branco - RR. ACTA Geográfica, Boa Vista, 35 (14), p. 48-63

SANTOS, S. L. D. X.; CARVALHO, E. B. (2018). Solução alternativa coletiva simplificada de tratamento de água destinada ao consumo humano em pequenas comunidades. (Nota informativa). Brasília: Funasa/Departamento de Saúde Ambiental/Coordenação de Controle da Qualidade da Água para Consumo Humano e Suest Pará. 

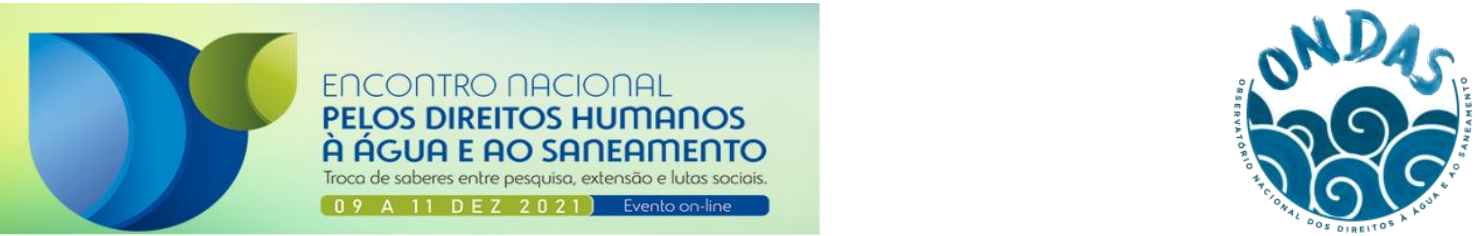

SEABRA, G. F. Dinâmica socioambiental no Baixo Rio Branco, estado de Roraima, Brasil.

Brazilian Geographical Journal: Geosciences and Humanities research medium, Ituiutaba, v.10, n.2, 2019, p. 112-130. 